\title{
Hidden Defects and Unexpected Properties of Graphene - How Advanced TEM Contributes to Materials Development
}

Benjamin Butz ${ }^{1,8}$, Christian Dolle ${ }^{1}$, Florian Niekiel ${ }^{1}$, Erdmann Spiecker ${ }^{1}$, Konstantin Weber $^{2}$, Bernd Meyer $^{2}$, Daniel Waldmann 3 , Ferdinand Kisslinger ${ }^{3}$, Heiko B. Weber ${ }^{3}$, Sam Shallcross ${ }^{4}$, Christian Halbig $^{5}$, Siegfried Eigler ${ }^{5,6}$ and Colin Ophus ${ }^{7}$

1. Institute of Micro- and Nanostructure Research \& Center for Nanoanalysis and Electron Microscopy (CENEM), Friedrich-Alexander-Universität Erlangen-Nürnberg, Erlangen, Germany.

2. Interdisciplinary Center for Molecular Materials (ICMM) \& Computer Chemistry Center (CCC), Friedrich-Alexander-Universität Erlangen-Nürnberg, Erlangen, Germany.

3. Chair for Applied Physics, Friedrich-Alexander-Universität Erlangen-Nürnberg, Erlangen, Germany.

4. Lehrstuhl für Theoretische Festkörperphysik, Friedrich-Alexander-Universität Erlangen-Nürnberg, Erlangen, Germany.

5. Department of Chemistry and Pharmacy and Central Institute of Materials and Processes (ZMP), Friedrich-Alexander-Universität Erlangen-Nürnberg, Erlangen, Germany.

6. Institut für Chemie und Biochemie, Organische Chemie, Freie Universität Berlin, Erlangen, Germany.

7. Molecular Foundry/National Center for Electron Microscopy, Lawrence Berkeley National Laboratory, Berkeley, CA, United States.

8. Visiting Scholar, Materials Science \& Engineering, Stanford University, Stanford, CA, United States.

Graphene and its chemically modified derivatives have widely been studied for more than a decade. Nonetheless, this highly interdisciplinary field, interdigitating with research on other (quasi-2D) layered materials like the chalcogenides, bears unexpected electronic, optical, chemical and mechanical properties almost every day. To understand novel properties and prospectively tailor functionality for application, it is indispensable to correlate to the material's local structure, chemistry and defects.

We will present outstanding examples, how state-of-the-art transmission electron microscopy, being highly sensitive to the material's crystal and atomic structure, its chemistry and even its topography down to the nm-scale, contributes to fundamental research as well as to material development:

i) Graphene from highly intact oxo-functionalized graphene (graphene oxide): We present a scalable route to prepare highly intact monolayer graphene oxide from graphite and its gentle reduction by low-energy electrons (Fig. 1, [1]).

ii) Linear magnetoresistance of $\mathrm{SiC}$ graphene: Basal-plane partial dislocations, occasionally named "strain solitons", introduce a change of the local stacking order of bi-/few-layer graphene and may consequently cause a unique degree of disorder such as in $\mathrm{SiC}$ graphene. Each dislocation's strain field alters the local electronic properties of the material, which explains the perfect linear magnetoresistance up to field strengths of more than $60 \mathrm{~T}$ (Fig. 2, [2-4]).

iii) Nanoscale topography of freestanding graphene membranes: Non-destructive dark-field TEM tomography allows us to determine the topography of crystalline membranes with exceptional nm-resolution. Together with complementary TEM (membrane thickness, crystal structure, defects, impurities), this provides comprehensive insight to understand, e.g., the mechanical properties (strain relaxation, plasticity, energy dissipation) of such thin membranes and resonators. 


\section{References:}

[1] B Butz et al, Angew. Chem. Int. Edit. 55 (2016), pp. 15771

[2] B Butz et al, Nature 505 (2014), pp. 533

[3] F Kisslinger et al, Nat. Phys. 11 (2015), pp. 650

[4] D Waldmann et al, ACS Nano 7 (2013), pp. 4441

[5] The authors acknowledge financial support by the German Research Foundation (DFG) within the frameworks of the SFB953 Synthetic Carbon Allotropes, grants no. BU 2875/2-1 and no. EI 938/3-1, and the Erlangen Cluster of Excellence 315 Engineering of Advanced Materials.
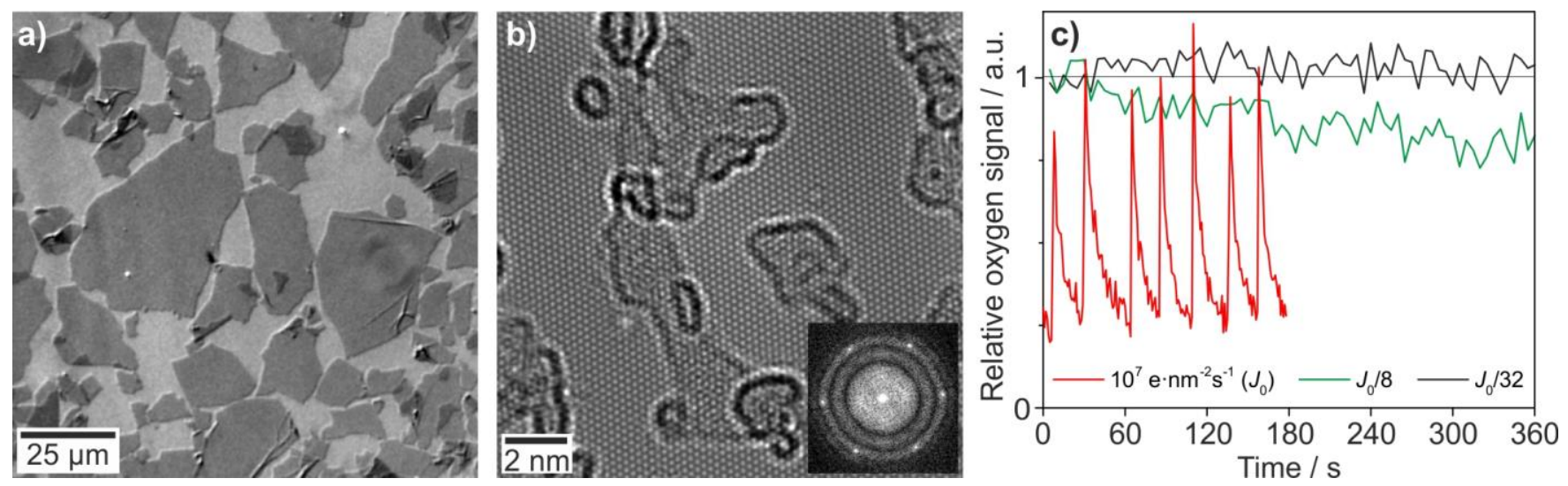

Figure 1. a) Optical micrograph of monolayer oxo-functionalized graphene flakes, b) integrity of underlying carbon framework of monolayer flake proven by aberration-corrected HRTEM, c) reduction of oxo-functionalized graphene by $80 \mathrm{kV}$ electrons (dependency on beam current density). (Ref. [1])
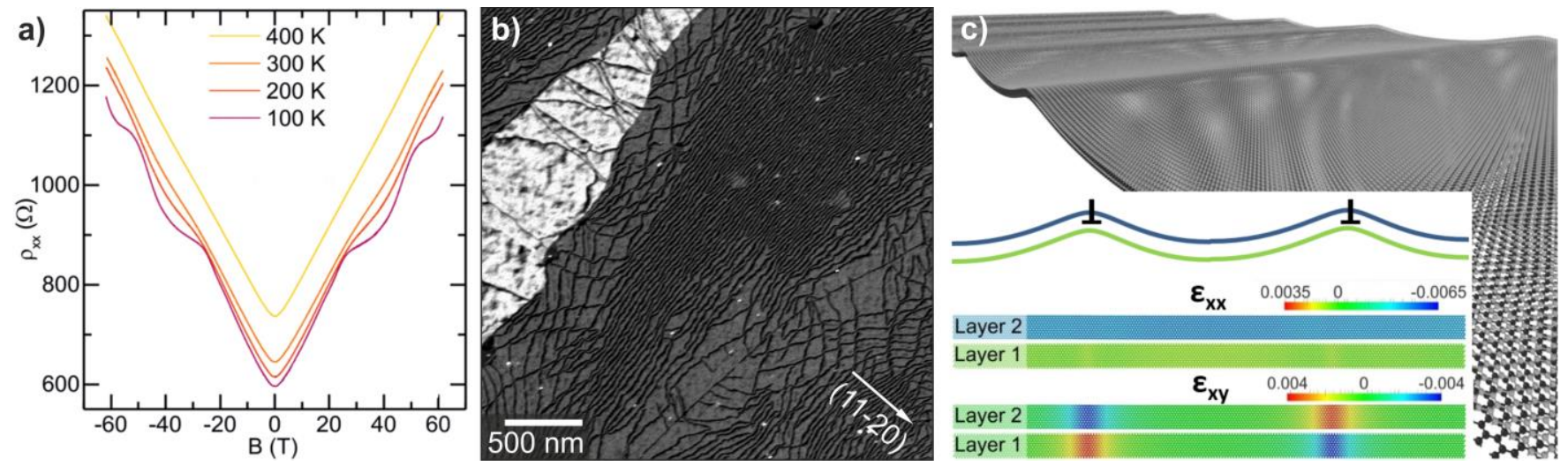

Figure 2. a) Perfect linear dependency of in-plane resistivity of $\mathrm{SiC}$ graphene on perpendicular magnetic field, b) dislocation network (dark lines) in bilayer and trilayer SiC graphene (DF-TEM micrograph), c) atomistic model of bilayer graphene topography at partial dislocation cores, inset: strain distribution as proven by dark-field TEM imaging. (Ref. [2-4]) 